\title{
Immunohistochemistry in formalin-gel fixed tissues
}

\author{
Amadeu Borges-Ferro; Ana Bastos Santos; Joana Louro Filipe \\ Author Affiliations: \\ *Corresponding author: amadeuferro@gmail.com \\ Department of Biomedical Sciences in Histocellular Pathology, Escola Superior de Tecnologia \\ da Saúde de Lisboa, Instituto Politécnico de Lisboa, Portugal \\ Keywords: Formalin gel, Fixation, Immunohistochemistry \\ DOI: http://dx.doi.org/10.1179/2046023614Y.0000000040
}

\section{ABSTRACT}

There are several hazards in histopathology laboratories and its staff must ensure that their professional activity is set to the highest standards while complying with the best safety procedures. Formalin is one of the chemical hazards to which such professionals are routinely exposed. To decrease this contact, it is suggested that $10 \%$ neutral buffered liquid formalin (FL) is replaced by $10 \%$ formalin-gel (FG), given the later reduces the likelihood of spills and splashes, and decreased fume levels are released during its handling, proving itself less harmful. However, it is mandatory to assess the effectiveness of FG as a fixative and ensure that the subsequent complementary techniques, such as immunohistochemistry (IHC), are not compromised. Two groups of 30 samples from human placenta have been fixed with FG and FL fixatives during different periods of time $(12,24$, and 48 hours) and, thereafter, processed, embedded, and sectioned. IHC for six different antibodies was performed and the results were scored (0-100) using an algorithm that took into account immunostaining intensity, percentage of staining structures, non-specific immunostaining, contrast, and morphological preservation. Parametric and non-parametric statistical tests were used (alpha $=0.05$ ). All results were similar for both fixatives, with global score means of $95.36 \pm 6.65$ for $\mathrm{FL}$ and $96.06 \pm 5.80$ for $F G$, and without any statistical difference $(P>0.05)$. The duration of the fixation had no statistical relevance also $(P>0.05)$. So it is proved here FG could be an effective alternative to $\mathrm{FL}$.

Keywords: Formalin gel, Fixation, Immunohistochemistry http://www.maneyonline.com/doi/abs/10.1179/2046023614Y.0000000040?journalCode=his 